UCRL-JC-130955

PREPRINT

\title{
A Blast Wave Diagnostic for the Petawatt Laser System
}

K. S. Budil, B. A. Remington, D. M. Gold, K. Estabrook, J. Kane, P. M. Bell, D. Pennington, C. Brown, M. H. Key, M. D. Perry

This paper was prepared for submittal to the 12th Topical Conference on High-Temperature Plasma Diagnostics Princeton, NJ

June 7-11, 1998

June 3, 1998

This is a preprint of a paper intended for publication in a journal or proceedings Since changes may be made before publication, this preprint is made available with the understanding that it will not be cited or reproduced without the permission of the author 


\section{DISCLAIMER}

This document was prepared as an account of work sponsored by an agency of the United States Government. Neither the United States Government nor the University of California nor any of their employees, makes any warranty, express or implied, or assumes any legal liability or responsibility for the accuracy, completeness, or usefulness of any information, apparatus, product, or process

disclosed, or represents that its use would not infringe privately owned rights. Reference herein to any specific commercial product, process, or service by trade name, trademark, manufacturer, or otherwise, does not necessarily constitute or imply its endorsement, recommendation, or favoring by the United States Government or the University of California. The views and opinions of authors expressed herein do not necessarily state or reflect those of the United States Government or the University of California, and shall not be used for advertising or product endorsement purposes. 


\section{A Blast Wave Diagnostic for the Petawatt Laser System*}

K. S. Budil, B. A. Remington, D M Gold, K Estabrook, J. Kane, P M. Bell, D. Pennington, C Brown, M H Key, and M. D Perry Lawrence Livermore National Laboratory, Livermore, CA 94550

Abstract

We report on a diagnostic to measure the trajectory of a blast wave propagating through a plastic target $400 \mu \mathrm{m}$ thick. This blast wave is generated by the irradiation of the front surface of the target with $\sim 400 \mathrm{~J}$ of $1 \mu \mathrm{m}$ laser radiation in a $20 \mathrm{ps}$ pulse focused to $\mathrm{a} \sim 50 \mu \mathrm{m}$ diameter spot, which produces an intensity in excess of $10^{18} \mathrm{~W} / \mathrm{cm}^{2}$. These conditions approximate a point explosion and a blast wave is predicted to be generated with an initial pressure nearing 1 Gbar which decays as it travels approximately radially outward from the interaction region We have utilized streaked optical pyrometry of the blast front to determine its time of arrival at the rear surface of the target Applications of a self-similar Taylor-Sedov blast wve solution allows the amount of energy deposited to be estimated The experiment, LASNEX design simulations and initial results are discussed. *This work was performed under the auspices of the U. S. Department of Energy by the Lawrence Livermore National Laboratory under contract number W-7405-ENG-48 


\section{Introduction}

The universe is filled with examples of strong, radiative shock waves interacting with their surroundings A common example in astrophysics is the explosion of a supernova wherein a shock wave propagates radially outward from the collapsed core of the progenitor star. In this expanding blast wave radiative effects are manifested in two ways. Radiation leaving the hot shocked plasma cools this region and acts as a radiative precursor which preheats the plasma in front of the shock. This effect is illustrated in one dimension in Figure 1. The detailed evolution of such a system becomes even more complex when examined in two dimensions. Depending on the Mach number $\left(\mathrm{M}=\mathrm{v}_{\text {shock }} / \mathrm{c}_{\text {sound }}\right)$ of the shock and the rate of radiative cooling present as defined by the cooling curve $\left(\Lambda=\Lambda_{0} T^{\alpha}\right.$, where $n^{2} \Lambda$ is ergs $/ \mathrm{cm}^{3}$-sec of cooling), the shock front is susceptible to instabilities.[1] Laboratory experimental tests of the models of radiative shocks are difficult to construct since the plasma must be very hot $\left(\mathrm{T}_{\mathrm{e}} \sim 1 \mathrm{keV}\right)$ in order for radiative effects to significantly alter the hydrodynamic evolution of the system.

However, a new generation of short pulse lasers,[2] capable of generating intensities in excess of $10^{18} \mathrm{~W} / \mathrm{cm}^{2}$, has allowed us to begin investigating the properties of radiative shock waves in detail. At such intensities, the temperature in the hot spot of the plasma generated by the laser-solid target interaction is believed to be in excess of $1 \mathrm{MeV}$ and the 
pressure is predicted to be $\sim 1$ Gbar.[3] Detailed simulations of the laser-target interaction indicates that initially a plasma is generated at the surface which gives rise to a burst of hot $(\mathrm{MeV})$ electrons which propagate through the target in a forward-directed cone with a roughly $30^{\circ}$ opening angle.[4] These hot electrons account for $\sim 30 \%$ of the energy deposited by the laser pulse. Another $\sim 30 \%$ of the incident energy is radiated away from the surface plasma. Over several hundred picoseconds, the remainder of the laser energy is hydrodynamically coupled into the solid and a blast wave is launched. Because of the small size of the spot into which the laser energy is deposited, it is well-approximated by a point explosion and the blast wave settles into an adiabatic expansion described by the self-similar Sedov solution where the position of the blast wave is given by

$$
r_{\text {blast }}(t)=f(\gamma) E^{1 / 5} t^{2 / 5}
$$

where $f(\gamma)$ is a coefficient dependent upon the material equation of state, $E$ is the energy deposited and $t$ is time.[5]

The interaction is illustrated in Figure 2 The trajectory of the spherically-expanding blast wave is a direct measure of the energy deposited within the laser spot. Any deviation from a smooth, roughly spherical front may be a measure of the energy deposited into the forward jet of hot electrons as well as an indication of instabilities on the blast front. Therefore, this diagnostic is potentially a unique, direct probe of the partition of energy in the initial interaction. 


\section{Experimental Configuration and Data}

The blast wave studied herein was generated by focusing the pulse generated by the Petawatt laser system[2] onto the surface of a solid plastic target. The experimental schematic is shown in Figure 3. The incident laser pulse was $400 \mathrm{~J}$ at $1 \mu \mathrm{m}$ wavelength and had a temporal duration of $20 \mathrm{psec}$. The pulse was focused to $\mathrm{a} \sim 50 \mu \mathrm{m}$ spot by an $\mathrm{f} / 3$ parabola resulting in an intensity of approximately $1 \times 10^{18} \mathrm{~W} / \mathrm{cm}^{2}$. The target consisted of a $20 \mu \mathrm{m}$ $\mathrm{CH}$ ablator backed by $0.5 \mu \mathrm{m}$ of aluminum (utilized for a spectroscopic diagnostic) and finally $400 \mu \mathrm{m}$ of deuterated polystyrene $\left(\mathrm{CD}_{2}\right)$. An f/10 Cassegrain telescope (Questar QM1) placed $1 \mathrm{~m}$ from the target images the rear surface of the target.

The first attempt at a blast wave measurement wherein the image from

- the telescope was cast directly onto the slit of an optical streak camera placed on a direct line-of-sight to the target was unsuccessful due to a high background level from the copious amounts of hard x-ray and hot electrons produced by the laser-target interaction. The configuration was modified to remove the streak camera from proximity to the target chamber by constructing a $10 \mathrm{ft}$ vertical periscope and a 2-inch-thick blanket of lead was installed between the target chamber and the detector. Thus, any high energy photons or electrons present pass through the first turning mirror in the 
periscope and leave the system. The observing bandwidth of the optical system is centered around $\sim 350 \mathrm{~nm}\left(\mathrm{~T}_{\mathrm{r}} \sim 3 \mathrm{eV}\right)$.

Figure 4(a) shows a raw image of the data and an averaged lineout through this image is contained in Figure 4(b). The data show two interesting features. First a weak prompt signal is observed which is correlated to the arrival of the intense laser pulse at the surface of the target. This is probably caused by the initial burst of hot electrons rapidly heating the rear surface of the target to several $\mathrm{eV}$, a very small temperature rise relative to the temperature at the front surface plasma. After $4.9 \mathrm{~ns}$ a very strong signal, which saturated the streak camera, is observed due to the breakout of the blast wave from the rear surface of the target. Another interesting feature is the lower intensity signal which precedes the blast wave by several hundred picoseconds. This may be the signal of the precursor caused by radiation from behind the blast front. Because of the high signal level, no definitive - determination of the shape of the breakout can be made.

\section{Theory and Simulations}

Figure 5 shows the results of a LASNEX[6] simulation of this experiment.[7] The density profiles shown in Figure 5(a) show that by $\sim 0.5 \mathrm{~ns}$ the hydrodynamic blast wave has formed and begun to propagate through the target. It reaches the rear surface at $\sim 5 \mathrm{~ns}$, in agreement with the observations. The radiation temperature profiles contained in Figure 5(b) 
show a strong radiative precursor. The simulations predict that the temperature at the rear surface of the target will exceed $3 \mathrm{eV}$ within the first $05 \mathrm{~ns}$ due to radiative preheat and that the temperature then drops back below an observable level as the blast propagates adiabatically through the target. Again at late times a small radiative precursor develops which reaches the rear surface a few hundred picoseconds in advance of the main blast, in reasonable agreement with the observations.

Since we have only experimentally measured a single point along the trajectory of the blast wave, we compare the results of the LASNEX simulations to the Sedov prediction. Figure 6(a) shows the blast wave trajectory from LASNEX as a function of time (solid circles) compared to the Sedov solution (solid line) where the prefactor $f(\gamma)$ has been adjusted to match the simulations. At times greater than $1 \mathrm{~ns}$ the blast wave appears to be evolving adiabatically. However, at earlier times the influence of the radiation field can be seen as shown in Figure 6(b). Here the time period from $0-1 \mathrm{~ns}$ is shown and the trajectory is plotted versus $\mathrm{t}^{2 / 5}$. Significant departures from the Sedov prediction are observed in this early stage indicating that perhaps the initial phase of the blast wave evolution is altered by radiation from behind the blast wave front.

\section{Conclusions and Future Directions}

We have made the first direct observation of the blast wave launched by the interaction of the Petawatt laser with a solid target. Such observations 
may provide a direct measure of how the laser energy deposited into the target is partitioned between hydrodynamic coupling, a forward-directed jet of hot electrons and energy radiated away from the hot surface plasma. Initial results are in reasonable agreement with LASNEX simulations. The LASNEX simulations have been compared with a Sedov solution for an adiabatically expanding blast wave and show good agreement except for early times when radiative effects may be important.

Future experiments will attempt to experimentally map the trajectory of the blast wave by varying the thickness of the target. Additionally, a portion of the Petawatt laser beam may be directed onto a secondary target to generate an $\mathrm{x}$-ray backlighter which may allow us to record a 2-dimensional image of the blast wave in flight. Deviations from a spherical shape may be indicative of the influence of the hot electron jet or the presence of instabilities on the blast wave front.

VI. Acknowledgments

The authors wish to acknowledge many fruitful discussions with S. Hatchett and the expert technical assistance of the operations staff at the Nova laser facility. 
[1] B. Draine and C. McKee, Ann. Rev. A\&A 31, 373 (1993); Science 252, 397 (1991); J. M. Shull and C. F McKee, Ap. J 227, 131 (1979); Strickland and J. Blondin, Ap. J 449, 727 (1995); J. Grun et al., Phys. Rev. Lett. 66, 2738 (1991).

[2] M. D Perry, Science and Technology Review, Publ. LLNL UCRL-54000-9612, P. 3.

[3] M. H. Key et al., Phys. Plasmas 5, 1966 (1998)

[4] S. Wilks ?? K. Wharton??

[5] Ya. B. Zel'dovich and Yu. P. Raizer, Physics of Shock Waves and HighTemperature Hydrodynamic Phenomena, edited by W. D. Hayes and R. F. Probstein (Academic Press, San Diego, 1966), vol 1, P 94.

[6] G. B. Zimmerman and W. L. Kruer, Comments Plasma Phys. Controlled Fusion 2, 51 (1975)

[7] K. Estabrook et al., in the proceedings of the 2 nd International Workshop of Laboratory Astrophysics with Intense Lasers, 19-21 March 1998, Tucson, AZ.

\section{- Figure Captions}

Figure 1. Diagram of the propagation of a radiative shock wave showing the effect of the radiation on the temperature, density and velocity of the shock front.

Figure 2: Illustration of the interaction of the high-intensity laser pulse with the solid target and subsequent hot electron and blast wave propagation. $E_{1}$ denotes energy hydrodynamically-coupled into the target in the form of a 
blast wave and $\mathrm{E}_{2}$ denotes energy deposited into a forward-directed jet of hot electrons

Figure 3: Schematic of the experimental configuration for the Petawatt blast wave measurement

Figure 4 (a) Raw image of the data from the streaked optical pyrometer (b) Averaged lineout through the image showing intensity in arbitrary units versus time.

Figure 5: LASNEX simulations of the (a) density $\left(\mathrm{g} / \mathrm{cm}^{3}\right)$ and (b) radiation temperature $(\mathrm{keV})$ as a function of depth in the target at a variety of times

Figure 6: (a) Comparison of the blast wave trajectory as a function of time - predicted by LASNEX to the self-similar Sedov solution. (b) Here the predicted blast wave trajectory for the first $1 \mathrm{~ns}$ of its evolution is plotted versus $\mathrm{t}^{2 / 5}$ showing significant deviations from the Sedov prediction at early times. 


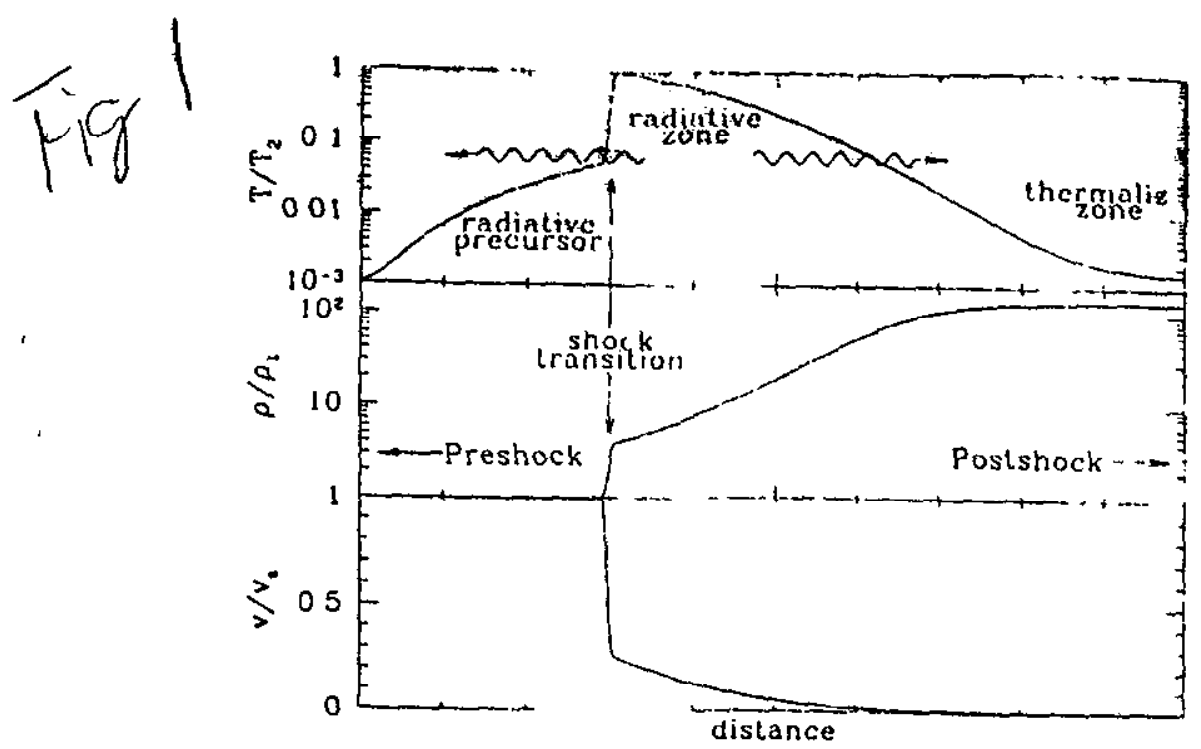

Figwe I Schematic: structure of a siring (xingle-fluid) shock wave showing temperature $r$ tensity $\rho_{1}$ and velocity $n$ (relative (n tiv shuck front) $\rho_{1}$ is the preshock density and $T_{2}$ is the temperature just behind the shock transition 


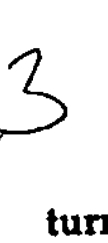

streak camera w/CCD readout

turning mirror

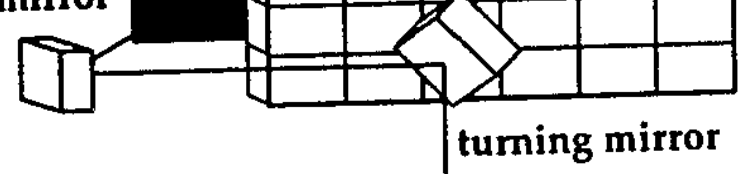

2 inch thick $\mathrm{Pb}$ shielding

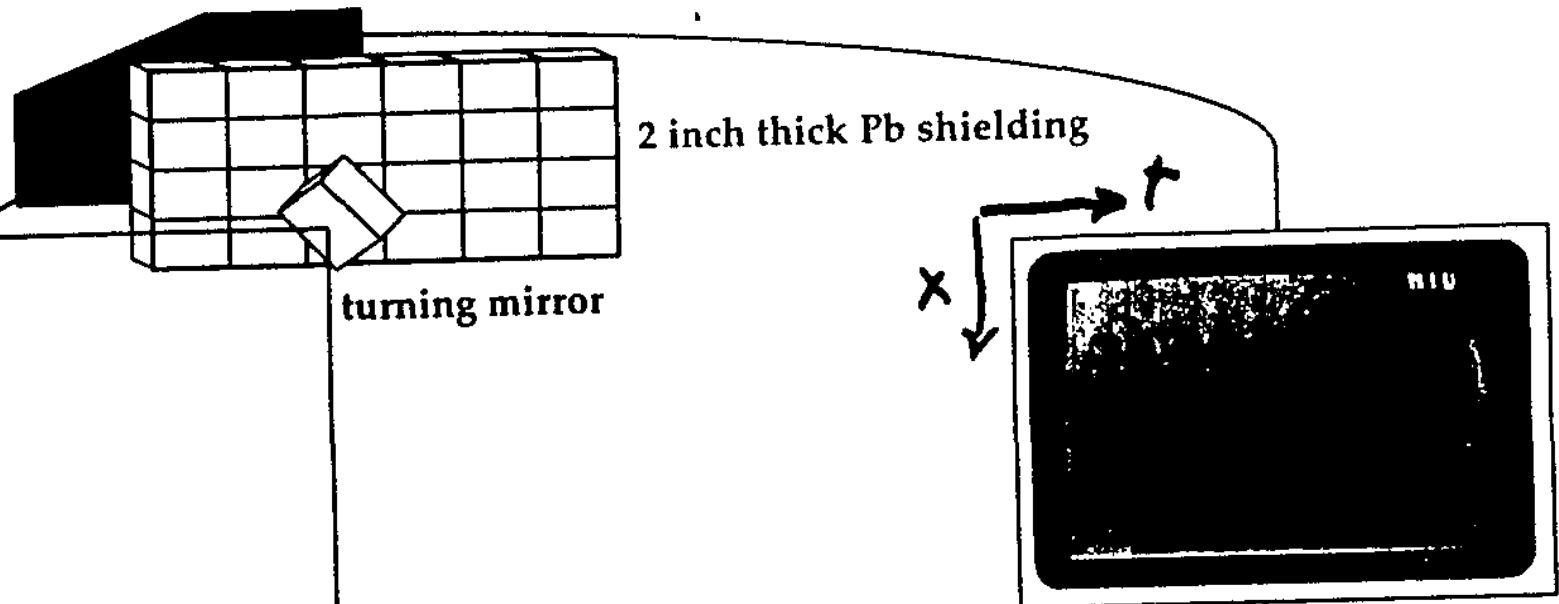

$\sim 10 \mathrm{ft}$. vertical periscope

Nova control system collects and displays data.

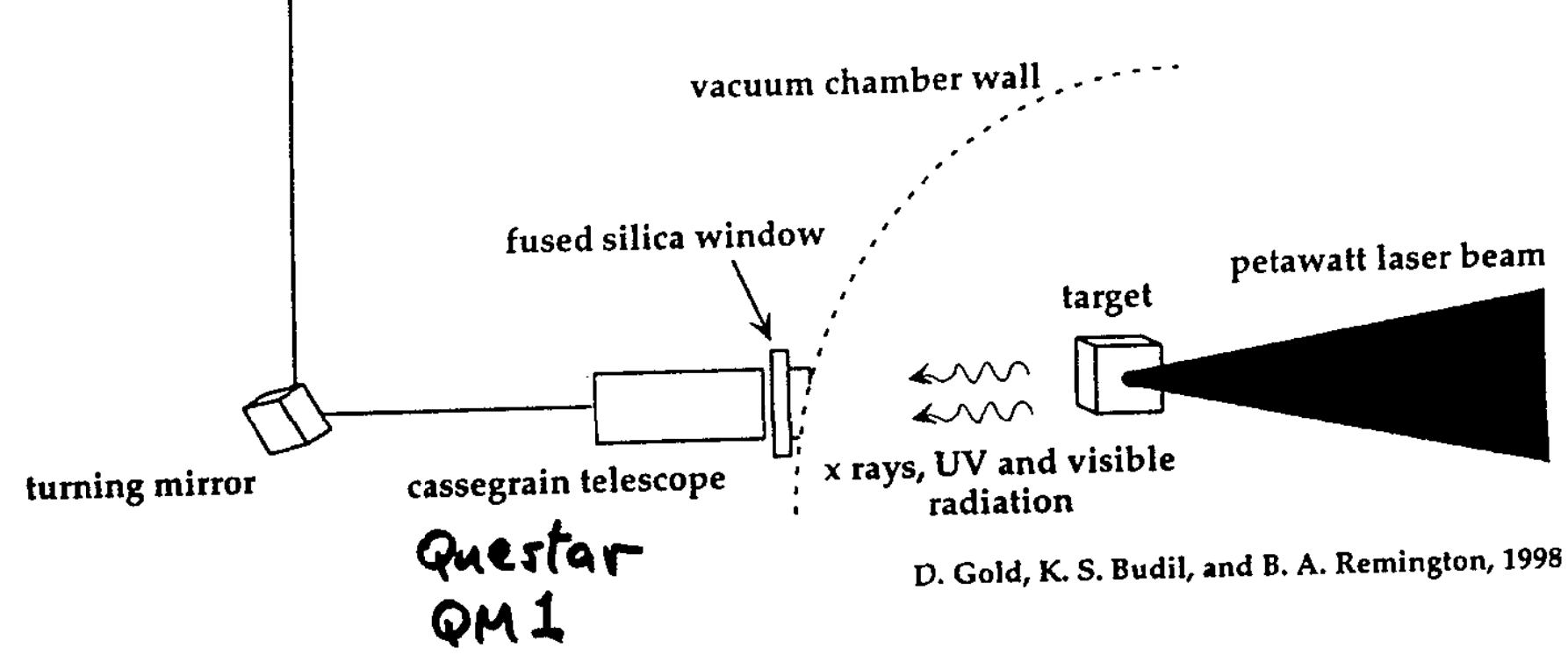



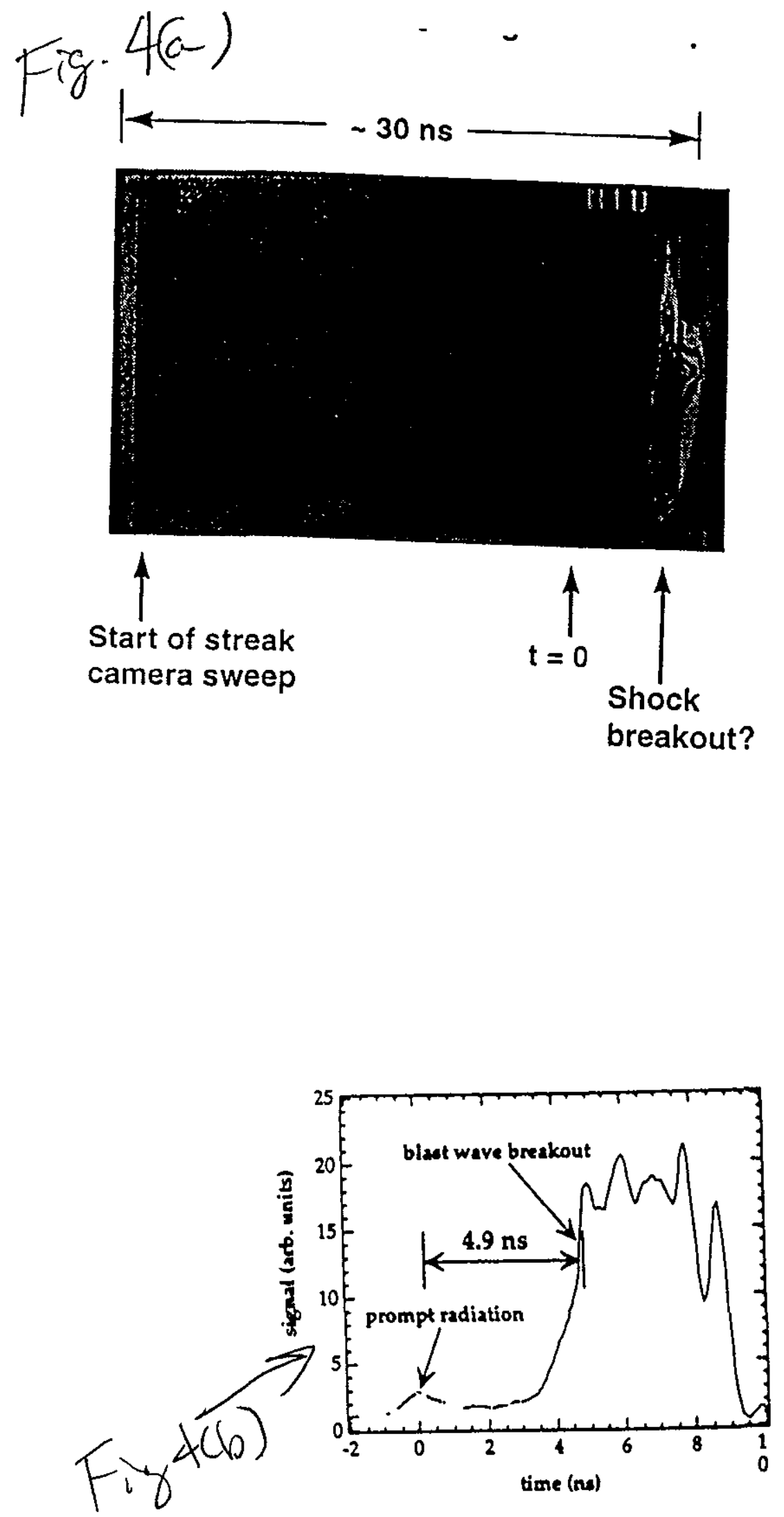

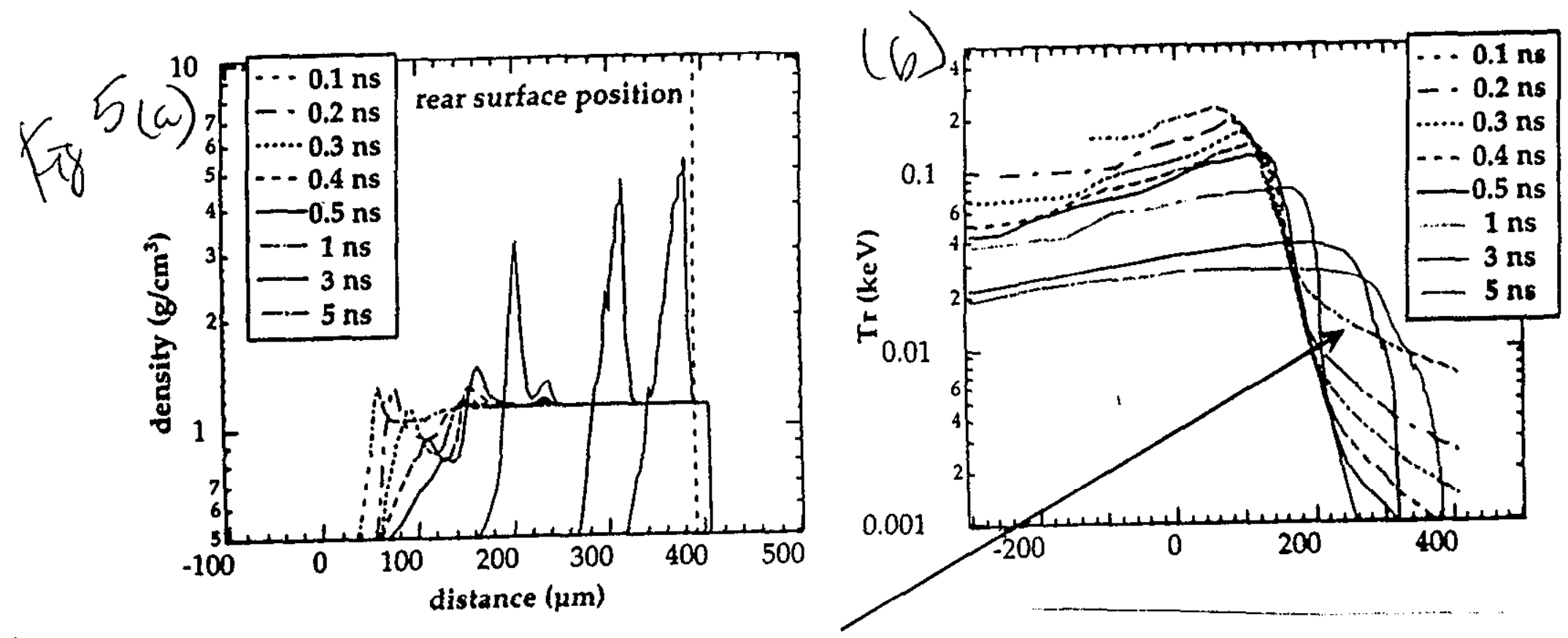

early time radiative precursor 


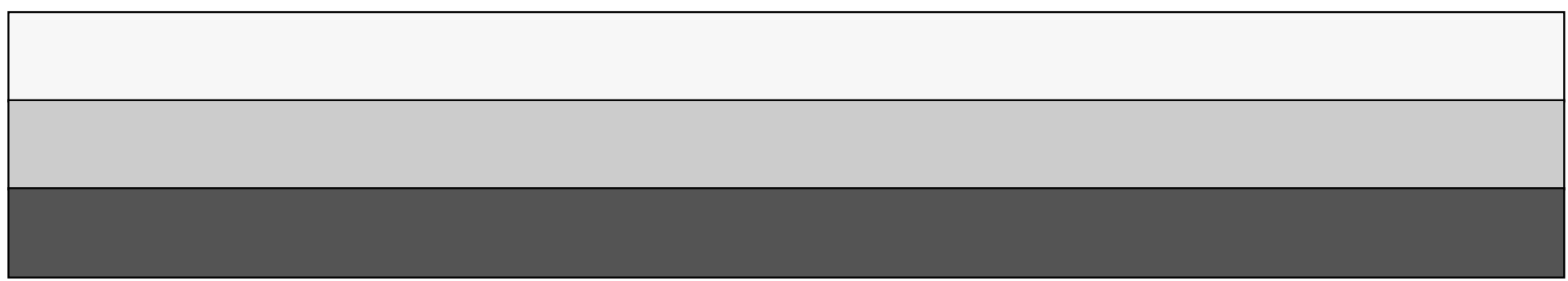

